Proceedings of the European Conference Physics of Magnetism 2011 (PM'11), Poznań, June 27-July 1, 2011

\title{
Electronic Structure and Magnetic Properties of the UPdAs$s_{2}$ Compound
}

\begin{abstract}
M. WERWIŃSKI* AND A. SzAJEK
Institute of Molecular Physics, Polish Academy of Sciences, M. Smoluchowskiego 17, 60-179 Poznań, Poland

The electronic band structure of UPdAss is calculated using full potential linearized augmented plane wave implemented in the WIEN2k code. Calculations were started with the exchange-correlation potential in the form proposed by Perdew, Burke and Ernzerhof (PBE). We additionally studied $\mathrm{PBE}+U$ approach with the Coulomb repulsion energies $U$ applied to the uranium $5 f$ orbital and varying from 0 to $4 \mathrm{eV}$. $\mathrm{PBE}+\mathrm{OP}$ with orbital polarization term was one more tested approach. To reproduce magnetic sequence ++-- a doubled in $c$ axis supercell is built. Initial magnetic moments on inequivalent uranium atoms are assumed to be opposite. The antiferromagnetic ground state is confirmed by comparison of total energies calculated for various magnetic configurations. Results of $\mathrm{PBE}+U(1.0 \mathrm{eV})+J(0.5 \mathrm{eV})$ and $\mathrm{PBE}+\mathrm{OP}$ approaches are in the best agreement with the neutron scattering measurements of magnetic moments. The calculated total magnetic moment on uranium atoms is predicted to be equal to $1.47 \mu_{\mathrm{B}}$
\end{abstract}

PACS: 71.20.-b, 75.25.-j

\section{Introduction}

$\mathrm{UPdAs}_{2}$ [1] together with $\mathrm{UNiAs}_{2}[2,3], \mathrm{UCuAs}_{2}$ [4] and $\mathrm{UCoAs}_{2}[5]$ belongs to a group of arsenides ternaries $\mathrm{UTAs}_{2}$ which is a part of a wider pnictides family $\mathrm{UTX}_{2}$ (where $\mathrm{X}=\mathrm{P}, \mathrm{As}, \mathrm{Sb}, \mathrm{Bi}$ ) [6, 7]. The wide range of properties in these ternary compounds is related to uranium $5 f$-electrons, which show an intermediate character between the localized $4 f$ and itinerant $3 d$-electrons system. Less significant are $d$-electrons from the transition metal $\mathrm{T}$ and $p$-electrons from a pnictogen $\mathrm{X}$.

The crystallographic and magnetic data of $\mathrm{UPdAs}_{2}$ compound were reported by Murasik et al. [1]. Preliminary magnetic measurements have shown that compound orders antiferromagnetically below $240 \mathrm{~K}$. A subject of the neutron diffraction studies was a polycrystalline sample. It has been shown that magnetic moments are oriented along the $c$ axis and localized on uranium atoms. Measurements at $8.7 \mathrm{~K}$ yield to $1.69 \pm 0.05 \mu_{\mathrm{B}}$ with sequence $++--[1,7]$. Previously, band structure for the isostructural $\mathrm{UCoAs}_{2}$ and $\mathrm{UNiAs}_{2}$ were reported $[8,9]$. In this paper, we present the results of $a b$ initio electronic structure calculations designed to reproduce the magnetic properties of $\mathrm{UPdAs}_{2}$ compound.

\section{Details of calculations}

To construct crystal structure we used experimental lattice parameters $(a=3.973(1) \AA, c=9.480(3) \AA)$ and Wyckoff positions [1]. To reproduce the sequence of magnetic moments orientation ++-- a supercell doubled along $c$ axis was built and the uranium atoms positions were split into two nonequivalent types U1 and U2 (see Fig. 1 and Table I). A space group of the supercell has

\footnotetext{
* corresponding author; e-mail: werwinski@ifmpan.poznan.pl
}

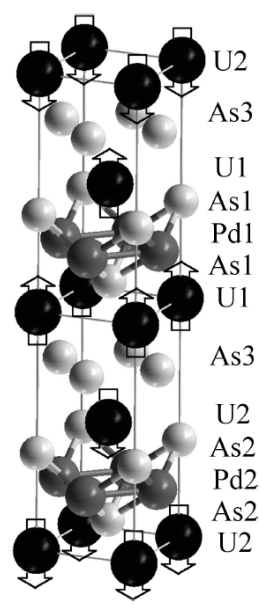

Fig. 1. The crystallographic and magnetic structure of $\mathrm{UPdAs}_{2}$.

TABLE I

The atomic positions of UPdAss. The antiferromagnetic supercell based on the experimental crystallographic data [1].

\begin{tabular}{c|c|c|c}
\hline \hline Atom & $x$ & $y$ & $z$ \\
\hline U1 (2c) & 0.25 & 0.25 & 0.36875 \\
U2 (2c) & 0.25 & 0.25 & 0.86875 \\
Pd1 (2b) & 0.25 & 0.75 & 0.5 \\
Pd2 (2a) & 0.25 & 0.75 & 0.0 \\
As1 (2c) & 0.25 & 0.25 & 0.59135 \\
As2 (2c) & 0.25 & 0.25 & 0.09135 \\
As3 (4f) & 0.25 & 0.75 & 0.25
\end{tabular}

not changed and remained $P 4 / \mathrm{nmm}$. The antiferromagnetic solution was not assumed in advance. Seven inequivalent atoms forming the supercell were treated as separate types. The magnetic sequence ++-- were initiated by setting opposite signs of an initial spin split- 
ting on uranium atoms. It allowed the system to reach an antiferromagnetically ordered ground state.

We used the full-potential linearized augmented plane-wave (FP-LAPW) method as implemented in the WIEN2k code [10] within the generalized gradient approximation (GGA) for the exchange-correlation potential in the Perdew, Burke, Ernzerhof form (PBE) [11]. Relativistic effects were included with the second variational treatment of spin-orbit coupling. Calculations were performed for $612 k$-points in the irreducible wedge of the Brillouin zone $(33 \times 33 \times 7$ mesh $)$ and a plane wave cut-off parameter of $R K_{\mathrm{MAX}}=8$. The total energy convergence criterion was $10^{-6} \mathrm{Ry}$.

Two approaches: the $\mathrm{PBE}+U$ (introduced by Anisimov et al. [12] for LSDA (local spin density approximation)) and so-called orbital polarization (OP) term (as proposed by Brooks [13] and Eriksson et al. [14]) were used for a better description of strong correlations and to lower discrepancy between calculated and experimental magnetic moments.

\section{Results and discussion}

The spin polarized calculations were performed for various initial spin splitting configurations. Solutions for ferro- and antiferromagnetic configurations were obtained. The latter one was more stable by about $0.01 \mathrm{eV}$ per formula unit.

\section{TABLE II}

The spin, orbital and total local magnetic moments $\mu$ $\left[\mu_{\mathrm{B}}\right]$ on $\mathrm{U} 1$ atom. The magnetic moments on U2 atom are exactly opposite.

\begin{tabular}{l|c|c|c}
\hline \hline \multicolumn{1}{c|}{ Method } & $\mu_{\mathrm{s}}$ & $\mu_{l}$ & $\mu_{\text {tot }}$ \\
\hline PBE & 1.27 & -1.81 & -0.54 \\
PBE $+U(1 \mathrm{eV})+J(0.5 \mathrm{eV})$ & 1.59 & -3.06 & -1.47 \\
PBE + OP & 1.51 & -2.98 & -1.47 \\
\hline Exp. [1] & \multicolumn{3}{|c}{$1.69(5)$}
\end{tabular}

The experimental value of magnetic moment on uranium atoms $\left(1.69(5) \mu_{\mathrm{B}}\right)$ is known from the neutron diffraction experiment [1]. PBE approach gives underestimated value $0.54 \mu_{\mathrm{B}} / \mathrm{U}$ atom (see Table II). Two series of calculations within the $\mathrm{PBE}+U$ approach were performed to improve the description of $5 f$ electrons. On-site Coulomb energy $U$ was varied from 0 to $4 \mathrm{eV}$ for the exchange interaction term $J$ equal to 0 and $0.5 \mathrm{eV}$ (see Fig. 2). In best agreement with experiment is magnetic moment calculated for approach $\mathrm{PBE}+U$ with values $U=1 \mathrm{eV}, J=0.5 \mathrm{eV}$. The PBE $+\mathrm{OP}$ formalism also improves results which are very similar to $\mathrm{PBE}+U(1 \mathrm{eV})+J(0.5 \mathrm{eV})$ and in good agreement with the neutron diffraction measurements (see Fig. 2).

Figures 3 and 4 present calculated densities of electronic states (DOS). The spin projected DOS plots of $\mathrm{U}(5 f)$ electrons are collected in Fig. 3. The $\mathrm{PBE}+U$ procedure causes shift of occupied $\mathrm{U}(5 f)$ states to higher binding energies and unoccupied towards energies above the Fermi level $\left(E_{\mathrm{F}}\right)$. DOS's obtained for

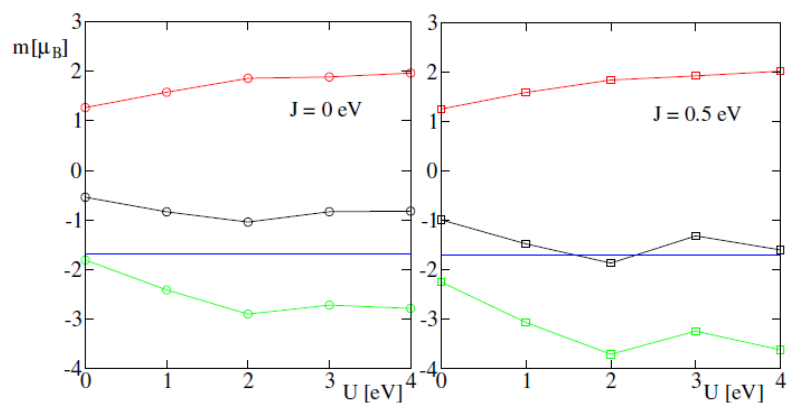

Fig. 2. Spin (red), orbital (green) and total (black) magnetic moments $\mu\left[\mu_{\mathrm{B}}\right]$ on uranium atom for UPdAs 2 depending on on-site repulsion $U$ for two values of $J$. Graph on the left for $J=0 \mathrm{eV}$, graph on the right for $J=0.5 \mathrm{eV}$. Blue line for experimental value $1.69(5) \mu_{\mathrm{B}}[1]$

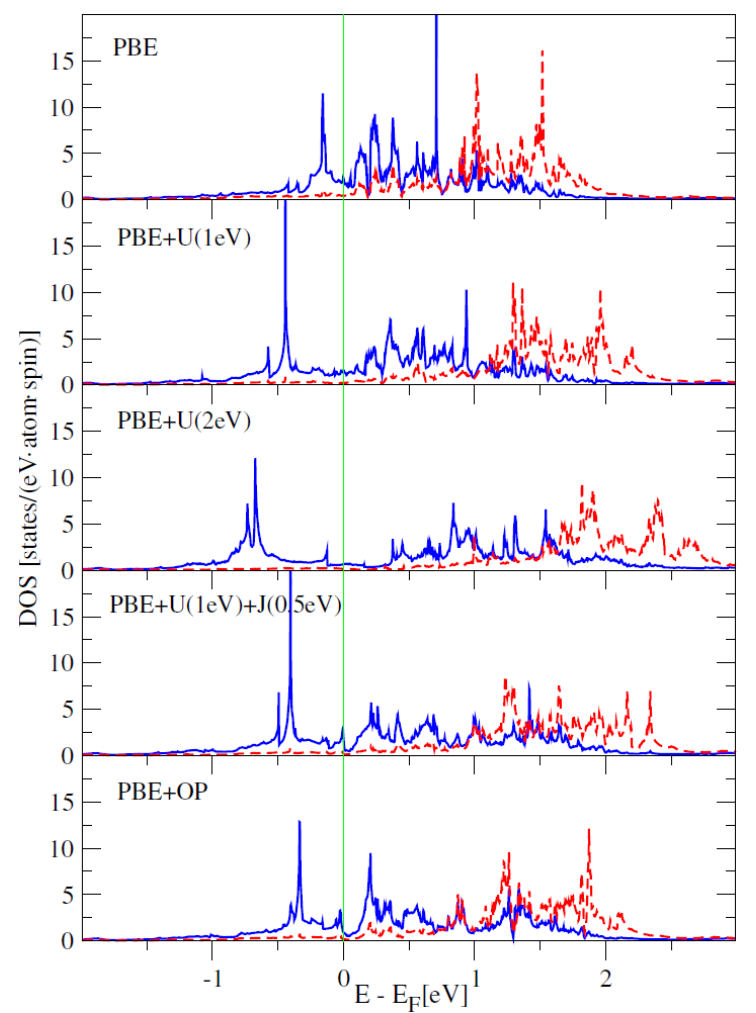

Fig. 3. Spin projected partial densities of states for uranium (U1) $5 f$-electrons of $\mathrm{UPdAs}_{2}$ compound calculated without and with orbital polarization corrections $(\mathrm{PBE}+\mathrm{OP})$ and using the $\mathrm{PBE}+U$ formalism. Solid blue curves for the majority spin and dashed red curves for the minority spin.

$\mathrm{PBE}+U(1.0 \mathrm{eV})+J(0.5 \mathrm{eV})$ and $\mathrm{PBE}+\mathrm{OP}$ approaches are similar. Presented in Fig. 4 total DOS for $\mathrm{PBE}+U(1.0 \mathrm{eV})+J(0.5 \mathrm{eV})$ are mainly a superposition of $\operatorname{Pd}(4 d)$ and $\mathrm{U}(5 f)$ states. Just above $E_{\mathrm{F}}$ a pseudogap is created. For $\mathrm{PBE}+\mathrm{OP} E_{\mathrm{F}}$ is located inside the pseudogap (see Fig. 3) from which comes a large reduction of calculated Sommerfeld specific heat coefficient $(\gamma)$ presented in Table III. Unfortunately, to our best knowl- 


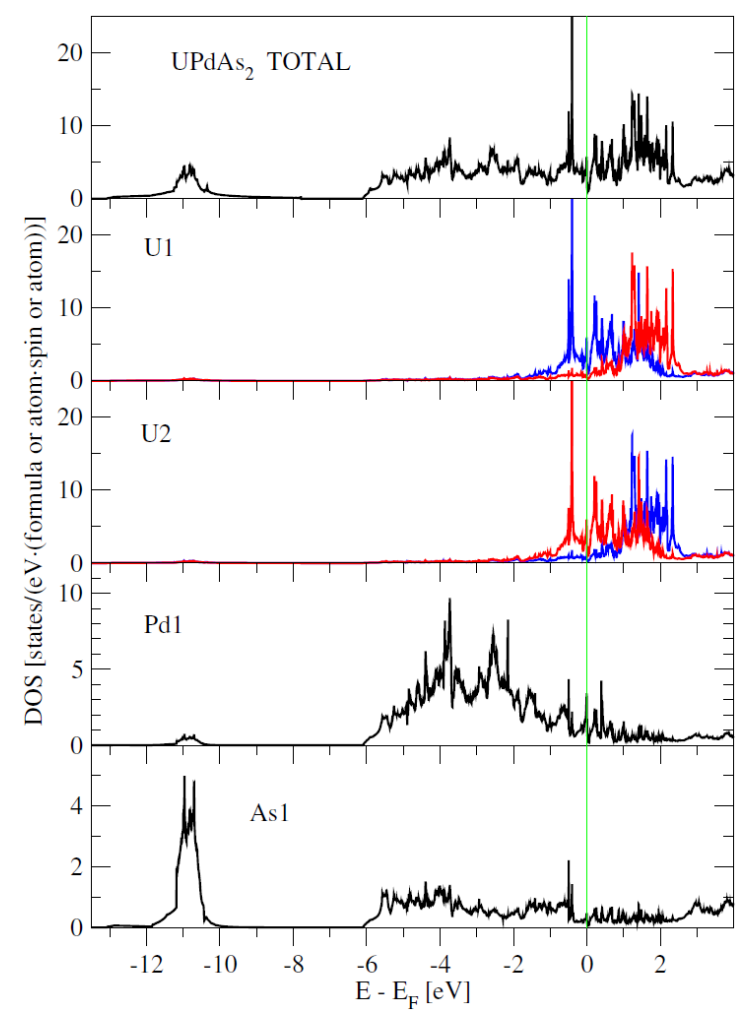

Fig. 4. Densities of states for $\mathrm{UPdAs}_{2}$ for

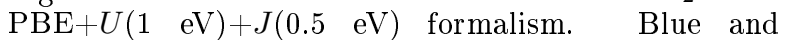
red curves for different spin types, black for DOS's without spin polarization.

edge, the experimental value of $\gamma$ is not available so we are not able to make a comparison.

TABLE III

Total densities of states on the Fermi level $\operatorname{DOS}\left(E_{\mathrm{F}}\right)$ [states/(eV (formula unit))] and calculated Sommerfeld specific heat coefficients $\gamma\left[\mathrm{mJ} /\left(\mathrm{mol} \mathrm{K}^{2}\right)\right]$.

\begin{tabular}{l|c|c}
\hline \hline & $\operatorname{DOS}\left(E_{\mathrm{F}}\right)$ & $\gamma$ \\
\hline $\mathrm{PBE}$ & 2.07 & 4.89 \\
$\mathrm{PBE}+U(1 \mathrm{eV})+J(0.5 \mathrm{eV})$ & 2.14 & 5.04 \\
$\mathrm{PBE}+\mathrm{OP}$ & 0.94 & 2.21
\end{tabular}

\section{Summary and conclusions}

In this paper we have presented results of the $a b i n i$ tio band structure calculations for the $\mathrm{UPdAs}_{2}$ compound. The FP-LAPW method was used within the $\mathrm{PBE}, \mathrm{PBE}+U$, and $\mathrm{PBE}+\mathrm{OP}$ formalism. The spin polarized calculations reproduced an antiferromagnetic solution with local magnetic moments on uranium atoms. A good agreement with an experimental result was obtained applying $\mathrm{PBE}+U(1 \mathrm{eV})+J(0.5 \mathrm{eV})$ and $\mathrm{PBE}+\mathrm{OP}$ formalisms.

\section{References}

[1] A. Murasik, P. Fisher, D. Kaczorowski, J. Phys., Condens. Matter 2, 3967 (1990).

[2] Z. Żołnierek, D. Kaczorowski, R. Troć, J. Less Common Met. 121, 193 (1986).

[3] P. Fischer, A. Murasik, D. Kaczorowski, R. Troć, Physica B 156, 829 (1989).

[4] J. Stępień-Damm, D. Kaczorowski, R. Troć, J. Less Common Met. 132, 15 (1987).

[5] D. Kaczorowski, H. Noël, M. Potel, J. Alloys Comp. 302, 1 (2000).

[6] D. Kaczorowski, J. Alloys Comp. 186, 333 (1992).

[7] D. Kaczorowski, R. Kruk, J.P. Sanchez, B. Malaman, F. Wastin, Phys. Rev. B 58, 9227 (1998).

[8] M. Werwiński, A. Szajek, Acta Phys. Pol. A 115, 244 (2009).

[9] M. Werwiński, A. Szajek, P. Leśniak, W.L. Malinowski, Acta Phys. Pol. A 118, 413 (2010).

[10] P. Blaha, K. Schwarz, G. Madsen, D. Kvasnicka, J. Luitz, WIEN2k, An Augmented Plane Wave + Local Orbitals Program for Calculating Crystal Properties, Karlheinz Schwarz, Techn. Universität Wien, Austria, 2001. ISBN 3-9501031-1-2.

[11] J.P. Perdew, K. Burke, M. Ernzerhof, Phys. Rev. Lett. 77, 3865 (1996).

[12] V.I. Anisimov, I.V. Solovyev, M.A. Korotin, M.T. Czyzyk, G.A. Sawatzky, Phys. Rev. B 48, 16929 (1993).

[13] M.S.S. Brooks, Physica B 130, 6 (1985).

[14] O. Eriksson, B. Johansson, M.S.S. Brooks, J. Phys. C 1, 4005 (1989). 